\title{
Uso de la orina humana como fertilizante en la producción de lechuga Waldmann green (Lactuca sativa $L$.)
}

\section{Use human urine as fertilizer in producing lettuce Waldmann green (Lactuca sativa L.)}

Mamani-Mamani Virginia ${ }^{1,2^{*}}$, Loza-Murguia Manuel $^{4,5}$, Coronel-Quispe Laoreano ${ }^{1,2}$, Sainz-Mendoza Humberto ${ }^{2,3}$ Paye-Huaranca Victor ${ }^{2}$, Coronel Felipe ${ }^{2}$

\begin{tabular}{|c|c|}
\hline Datos del Artículo & Resumen \\
\hline 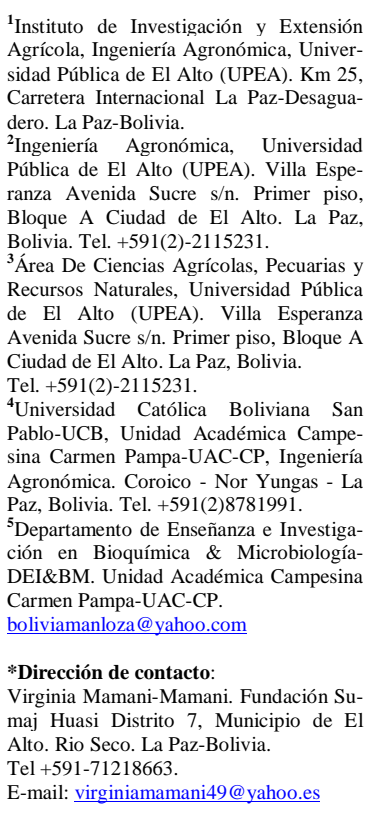 & $\begin{array}{l}\text { El objetivo del trabajo fue evaluar la respuesta del cultivo de lechuga, variedad Waldmann Green, a la aplicación } \\
\text { de la orina humana fermentada (OHF) en diferentes tiempos. La orina se obtuvo a partir de los baños ecológicos del } \\
\text { distrito } 7 \text { del municipio de El Alto. Estos exudados, se llevó a la fermentación durante diferentes tiempos: } 3,6 \text { y } 12 \\
\text { meses, con la finalidad de eliminar los posibles patógenos presentes. Los tratamientos aplicados fueron: T-1: sin orina, } \\
\text { T-2: tres meses de fermentación: T-3: seis meses de fermentación y T-4: doce meses de fermentación. El valor obtenido } \\
\text { de } 14.75 \mathrm{~cm} \text { de altura de planta, corresponde al tratamiento T-3, el testigo } 17.71 \mathrm{~cm} \text {. En el T3, se obtuvo un rendimien- } \\
\text { to de } 5.52 \mathrm{~kg} \mathrm{~m}^{-2} \text {. Este resultado podría deberse a la alta concentración de nitrógeno que tiene la orina humana y el } \\
\text { testigo presentó un rendimiento de } 3.04 \mathrm{~kg} \mathrm{~m}^{-2} \text {. Así mismo, se realizó el análisis bromatológico del producto para } \\
\text { evidenciar la presencia de posibles patógenos, los resultados no presentan presencia de patógenos como la Escherichia } \\
\text { coli, Salmonella spp }\end{array}$ \\
\hline \multirow{2}{*}{$\begin{array}{l}\text { Waldman Green, } \\
\text { orina humana fermentada, } \\
\text { Escherichia coli, } \\
\text { Salmonella spp. }\end{array}$} & (c) 2015. Journal of the Selva Andina Biosphere. Bolivia. Todos los derechos reservados. \\
\hline & Abstract \\
\hline $\begin{array}{l}\text { J Selva Andina Biosph. } \\
\text { 2015; 3(1):24-38. }\end{array}$ & The objective was to evaluate the response of growing lettuce, variety Waldmann Green, to the application of fermented \\
\hline $\begin{array}{l}\text { Historial del artículo } \\
\text { Recibido enero, } 2014 . \\
\text { Devuelto julio } 2014 \\
\text { Aceptado abril, } 2015 . \\
\text { Disponible en línea, mayo } 2015 \\
\end{array}$ & $\begin{array}{l}\text { ity. These exudates, fermentation took during different times: } 3,6 \text { and } 12 \text { months, in order to eliminate the possible } \\
\text { presence of pathogens. The treatments were T-1, with no urine, T-2, three months of fermentation, T- } 3 \text { six months of }\end{array}$ \\
\hline $\begin{array}{l}\text { Editado por: } \\
\text { Selva Andina Re- } \\
\text { search Society }\end{array}$ & $\begin{array}{l}\text { fermentations and T- } 4 \text { twelve months of fermentation. The highest value obtained was } 14.75 \mathrm{~cm} \text { plant height, which } \\
\text { corresponds to T-3 treatment and the control (T-1) reached } 17.71 \mathrm{~cm} \text {, plant height. The T-3 applied with six months of }\end{array}$ \\
\hline
\end{tabular}




\begin{tabular}{|c|c|}
\hline $\begin{array}{l}\text { Key words: } \\
\text { Waldman Green, } \\
\text { fermented human urine, } \\
\text { Escherichia coli, } \\
\text { Salmonella spp. }\end{array}$ & $\begin{array}{l}\text { obtained a performance of } 5.52 \mathrm{~kg} \mathrm{~m}^{-2} \text {. This result could be due to the high concentration of nitrogen that has human } \\
\text { urine and the witness presented a performance of } 3.04 \mathrm{~kg} \mathrm{~m}^{-2} \text {. Likewise, we realize product compositional analysis to } \\
\text { evaluate the presence of potential pathogens and according to the results did not present infestation of pathogens such as } \\
\text { Escherichia coli and Salmonella spp. It is therefore suitable for human consumption without presenting health risk. }\end{array}$ \\
\hline & (C) 2015. Journal of the Selva Andina Biosphere. Bolivian. All rights reserved. \\
\hline
\end{tabular}

\section{Introducción}

La orina humana se ha utilizado para diversos propósitos desde tiempos antiguos, últimamente empezó a ganar popularidad, ya que contiene altas concentraciones nutrientes para las plantas incluyendo el nitrógeno $(\mathrm{N})$, fósforo $(\mathrm{P})$ y potasio $(\mathrm{K})$, el contenido de $\mathrm{N}$ en la orina reportado en literatura oscila entre $700 \mathrm{mg} \mathrm{L}^{-1}$ a $900 \mathrm{mg} \mathrm{L}^{-1}, 500-650 \mathrm{mg} \mathrm{L}^{-1} \mathrm{P}$ y $\mathrm{K}$ 500-800mg L $\mathrm{L}^{-1}$ (Heinonen-Tanski \& WijkSijbesma 2005, Kirchmann \& Pettersson 1995).

El volumen de orina que es liberada del cuerpo humano varía de persona a persona y día a día siendo la media por persona de $1.5 \mathrm{~L}$ con fluctuaciones entre 1 y $2.5 \mathrm{~L}$ (Rauch et al. 2003), depende principalmente de la cantidad de líquido que bebe y transpira una persona Vinnerås et al. (2002), se puede esperar una concentración de 3 a 7 g de nitrógeno por litro de orina (Richert et al. 2011).

La orina inicialmente es estéril en el momento que abandona el cuerpo, pero hay varias interferencias que pueden contaminarla con microorganismos patógenos presentes en la materia fecal, infección de vías urinarias u otras enfermedades (Höglund 2001), por lo tanto debe de tener un tratamiento previo a su utilización para garantizar la inocuidad necesaria para emplearla en diversos cultivos. Los cálculos de riesgo han demostrado que en un período de un mes de espera la reducción del nivel de riesgo es impor tante y en combinación con otras barreras de la estrategia de barreras múltiples, el resultado será un riesgo muy por debajo de 10-6 AVAD para las bacterias patógenas, virus y protozoos parásitos (WHO 2006).

El tratamiento que se recomienda para poder utilizar la orina en la agricultura es el almacenamiento, debido que no se necesita mucha energía para su funcionamiento ni instrumentos para su procesamiento y se ha mostrado que los productos obtenidos de este tratamiento son altamente recomendados para su utilización en la agricultura como se ha mostrado en los casos de estudio de Tidåker et al. 2007a, AdeOluwa \& Cofie 2012, Pradhan et al. (2007), (Pradhan et al. 2009, 2010), Heinonen-Tanski et al. 2007, Shrestha et al. 2013. Este sistema consiste en separar la orina desde el origen (desviación de orina), colectarla en depósitos de mayor tamaño y almacenarla por varios meses. Esto tiene efecto inactivador en los patógenos, prolongar el almacenamiento es a menudo sugerido, siendo un adecuado método de higienización por lo que después de recibir este tratamiento se obtiene un fertilizantes a base de orina de alta calidad.

Por lo tanto el uso de fertilizante a base de orina podrían reducir la demanda de fertilizantes de la lechuga (Lactuca sativa L.) originaria de Asia e India en donde se cultiva en suelos areno-arcillosos, ricos en materia orgánica. En México se ha probado 
orina como fertilizante en lechuga de invernadero (Guadarrama et al. 2001). Los tratamientos compararon la orina con el compost, la mezcla de orinacompost y sin fertilizante. La dosis de aplicación fue de $150 \mathrm{~kg}$ de $\mathrm{N}$ total por hectárea en todos los tratamientos, excepto para el control sin fertilizar, dio mejor rendimiento en la lechuga, gracias a su alta disponibilidad de N. Las plantas con sistemas radiculares ineficientes o pequeños, por ejemplo, la zanahoria, la cebolla y la lechuga pueden beneficiarse de la aplicación repetida de orina durante todo el tiempo de cultivo (Thorup-Kristensen 2001).

La técnica de producción de lechuga con la fertilización de orina humana fermentada (exudado humano) es una opción para cultivar hortalizas en forma eficiente, competitiva, sana para el consumo de la población, además, permite acceder a formas de organización y gestión de la comunidad, generar procesos culturales de promoción personal, superación de la pobreza.

El trabajo se realizó con el propósito de desarrollar una alternativa en la producción de lechuga, variedad Waldmann green, con la fertilización de orina humana fermentada en diferentes tiempos, para mejorar la producción de alimentos y reducir la contaminación por excretas humanas en la zona periurbana de Villandrani, El Alto.

\section{Materiales y métodos}

El trabajo se realizó en los predios de la Fundación Sumaj Huasi, ubicado en la zona San Roque Villandrani, municipio de El Alto, a $16 \mathrm{~km}$ al este de la ciudad de La Paz, entre $16^{\circ} 28^{\prime} 60^{\prime \prime}$ latitud sur y $68^{\circ}$ 17' 06" longitud oeste, a una altitud de $3961 \mathrm{msnm}$, corresponde a la clasificación del altiplano, seco húmedo.
La incidencia de helada se presenta en los meses de mayo a agosto, con una temperatura promedio anual de $7.1^{\circ} \mathrm{C}$., con masas de aire frío provenientes del Norte, que causan olas de frío principalmente en verano e invierno, a una velocidad de viento de 9.7 $\mathrm{km} \mathrm{h}^{-1}$, con una precipitación pluvial que alcanza $613.1 \mathrm{~mm}$ por año, por los meses de septiembre a abril (SENAMHI 2008).

El experimento se realizó en la carpa solar de la Fundación Sumaj Huasi, las dimensiones de la infraestructura son las siguientes: es de doble agua o dos caídas con una superficie total de $66 \mathrm{~m}^{2}$, de 11 $\mathrm{m}$ de largo y $6 \mathrm{~m}$ de ancho. La estructura es de piedras, cemento, adobes y tijerales $2 \times 4$, listones $2 \mathrm{x}$ 2. El ambiente protegido tiene una pared alta de $2 \mathrm{~m}$ y las paredes bajas de sus laterales son de $1.50 \mathrm{~m}$ de alto, superficie útil de $60 \mathrm{~m}^{2}$. El material de cubierta del techo es de agrofilm de $250 \mu \mathrm{m}$.

Recolección y proceso de fermentación de la orina. La fundación SUMAJ HUASI implemento el proyecto denominado Saneamiento básico seco, en las zonas periurbanas de la ciudad de El Alto, que tiene por objetivo el manejo y uso responsable de las excretas humanas para convertirlas en abono sólido y líquido.

Para ello, instaló en cada hogar familiar un sistema de baño seco, con inodoros especiales para ese propósito en donde las excretas se depositan por separado. Las heces en un recipiente combinado con aserrín y la orina en otro contenedor, en este caso plástico de color amarillo de $20 \mathrm{~L}$ de capacidad o de mayor volumen, dependiendo del número de miembros por familia.

De los hogares día por medio, se recoge la orina a través de un vehículo que tiene rutas definidas $\mathrm{y}$, se lleva al depósito, donde se tiene recipientes de mayor de 5000 L de capacidad de color negro, herméticamente cerrados procediéndose a la fermentación 
por 3, 6, y 12, paralelamente se realizó los análisis de la orina, con el fin de evaluar el proceso de fermentación.

El personal encargado del manejo y manipulación de la orina, así como de las heces, utiliza toda la protección que exige la seguridad y salud ocupacional.

Se construyó 16 unidades de platabandas en 10 de febrero 2011, se utilizó las botellas plásticas de reciclado (PET), con una dimensión de $2 \mathrm{~m}$ de largo por $1.50 \mathrm{~m}$ de ancho, y una altura de $0.20 \mathrm{~m}$. En la preparación del sustrato se utilizó una relación de mezcla de: $50 \%$ de tierra negra, $30 \%$ de estiércol ovino y $20 \%$ de turba. Esta preparación sólo se utilizó para el testigo. Para los otros tratamientos, se utilizó una relación de mezcla de: $50 \%$ de tierra negra, $30 \%$ de turba y $20 \%$ de arena fina.

El trasplante en las platabandas se realizó el 01 de marzo de 2011, teniendo cuidado en elegir plantines vigorosos del mismo tamaño, para uniformizar el ensayo. La distancia entre surcos a $0.30 \mathrm{~m}$ y $0.25 \mathrm{~m}$ entre plantas, teniendo una densidad de 24 plantines por tratamiento.

Posteriormente se mezcló el sustrato para ser aplicado con la orina humana fermentada. La aplicación de la orina humana fermentada fue: i) primera fertilización se realizó antes de trasplante de la lechuga, con una solución (concentración) de 1:4; 1 litro de orina fermentada en diferentes tiempos, en $4 \mathrm{~L}$ de agua. ii) La segunda y la tercera fertilización, se realizó cada 15 días. Esta aplicación se hizo directamente sobre la superficie del suelo, cerca del cuello de la planta, con una combinación de 1 litro de orina fermentada en $4 \mathrm{~L}$ de agua por $1 \mathrm{~m}^{2}$.

Para determinar el efecto en el cultivo por la aplicación de los tratamientos, se utilizó el diseño completamente al azar, con 4 tratamientos y 3 repeticiones, con fertilización de orina humana fermentada en 27 diferentes tiempos. Las variables de respuestas estudiadas fueron: altura de la planta $(\mathrm{cm})$, número de hojas, longitud y ancho de la hoja $(\mathrm{cm})$, longitud de la raíz $(\mathrm{cm})$, rendimiento de lechuga $\left(\mathrm{kg} / \mathrm{m}^{2}\right)$ y el análisis económico.

Para determinar la altura de las plantas, se realizó la medición de 6 plantas al azar por unidad experimental, tomando en cuenta la longitud desde el cuello de la planta, hasta el ápice de la hoja superior, utilizando una regla graduada en milímetros. Para la evaluación del número de hojas, se tomaron 6 plantas al azar por tratamiento, También se hizo la medición del ancho de la hoja, tomando en cuenta las hojas basales. Para determinar el rendimiento de la lechuga, se cosechó las mismas y se pesó las muestras de cada tratamiento; luego, se procedió a transformar en $\mathrm{kg}$ por $\mathrm{m}^{2}$. El análisis económico, se realizó de acuerdo al método de los aspectos involucrados en la producción que permite conocer la rentabilidad del cultivo de lechuga, mediante costo-beneficio e ingreso neto.

\section{Resultados}

Variables agroclimáticas. Los ambientes atemperados presentaron fluctuaciones micro climáticas, de acuerdo a las horas luz del día. Las variables evaluadas fueron: temperatura y humedad al interior del ambiente. Entendiendo que estas variables interactúan directamente con el crecimiento y desarrollo de un determinado cultivo; sin dejar de considerar las otras variables.

Temperatura durante el desarrollo del cultivo. En la figura 1 , se evidencia que la temperatura mínima se registró los días 7, 9, 11, 13, 15 y 17 de enero, a horas 7:00 am; obteniendo lecturas promedio de 8 ${ }^{\circ} \mathrm{C}$. La temperatura máxima extrema se registró los 
días 29 y 31 de enero, con valores de 39 y $35^{\circ} \mathrm{C}$ respectivamente a horas 14:00 p.m. Los cambios bruscos (amplitud térmica) entre la temperatura máxima y mínima, no afectaron significativamente al crecimiento y desarrollo del cultivo, porque no se han observado alteraciones morfológicas visibles en las plantas. El comportamiento estuvo por encima de los $9.5^{\circ} \mathrm{C}$ y la máxima llegó hasta $35.5^{\circ} \mathrm{C}$.

Tabla 1 Temperaturas máximas y mínimas en el interior de la cubierta durante el desarrollo del cultivo

\begin{tabular}{ccc}
\hline Fecha & T $^{\mathbf{}} \mathbf{C}$ (Máx.) & T $^{\mathbf{0}} \mathbf{C}$ (Mín.) \\
\hline $\mathbf{0 7 / 0 2 / 2 0 1 1}$ & 30.9 & 8.3 \\
$\mathbf{1 4 / 0 2 / 2 0 1 1}$ & 33.2 & 9.5 \\
$\mathbf{2 1 / 0 2 / 2 0 1 1}$ & 29.7 & 9.2 \\
$\mathbf{2 8 / 0 2 / 2 0 1 1}$ & 35.5 & 8.2 \\
$\mathbf{0 7 / 0 3 / 2 0 1 1}$ & 28.9 & 8.9 \\
$\mathbf{1 4 / 0 3 / 2 0 1 1}$ & 29.5 & 8.5 \\
$\mathbf{2 1 / 0 3 / 2 0 1 1}$ & 32.7 & 9.5 \\
\hline
\end{tabular}

Las temperaturas: máxima y mínima en los meses de cultivo fueron homogéneas, obteniéndose como promedio una máxima de $31.48^{\circ} \mathrm{C}$ y una mínima de $8.87^{\circ} \mathrm{C}$. Estos rangos y variaciones en temperatura, no afectaron al crecimiento y desarrollo del cultivo, porque, no se observaron alteraciones morfológicas, ni deficiencias visibles en las plantas.

Humedad relativa (HR). Para determinar este parámetro en el interior del ambiente protegido, se utilizó un higrómetro analógico tipo reloj; los datos que registra este instrumento son de lectura directa. El comportamiento de esta variable se aprecia en la figura 2, registrándose fluctuaciones diarias sin muchas variaciones durante el periodo del experimento, alcanzando una máxima de $84 \%$ y mínima de 49.5 $\%$ de humedad relativa. La mayor humedad relativa se registró por las mañanas ( 8 a 10 h) y las mínimas a partir de horas 14:00 pm.

Tabla 2 Comportamiento de humedad relativa al interior del ambiente protegido

\begin{tabular}{ccc}
\hline Fecha & HR (Máx.) & HR (Mín) \\
\hline $\mathbf{0 7 / 0 2 / 2 0 1 1}$ & 78.25 & 38.33 \\
$\mathbf{1 4 / 0 2 / 2 0 1 1}$ & 81.13 & 46.39 \\
$\mathbf{2 1 / 0 2 / 2 0 1 1}$ & 85.30 & 48.29 \\
$\mathbf{2 8 / 0 2 / 2 0 1 1}$ & 82.55 & 49.23 \\
$\mathbf{0 7 / 0 3 / 2 0 1 1}$ & 80.22 & 50.31 \\
$\mathbf{1 4 / 0 3 / 2 0 1 1}$ & 83.56 & 48.19 \\
$\mathbf{2 1 / 0 3 / 2 0 1 1}$ & 82.59 & 50.22 \\
\hline
\end{tabular}

Tabla 3 Análisis de la orina humana fermentada (OHF) en diferentes tiempos

\begin{tabular}{ccccc}
\hline Parámetro & Unidad & 3 Meses & 6 Meses & 12 Meses \\
\hline $\mathrm{pH}$ & & 9.4 & 9.1 & 9.3 \\
Conductividad eléctrica & $\mathrm{mS} / \mathrm{cm}$ & 44 & 42 & 45 \\
Fósforo total & $\mathrm{Pmg} / \mathrm{L}$ & 311 & 322 & 5214 \\
Nitrógeno total & $\mathrm{mg} / \mathrm{L}$ & 440 & 4026 & 313 \\
Sodio & $\mathrm{mg} / \mathrm{L}$ & 3464 & 3656 & 3830 \\
Potasio & $\mathrm{mg} / \mathrm{L}$ & 1870 & 1845 & 1894 \\
Calcio & $\mathrm{mg} / \mathrm{L}$ & 3.7 & 2.7 & 1.3 \\
Magnesio & $\mathrm{mg} / \mathrm{L}$ & 0.42 & 0.52 & 0.29 \\
\hline
\end{tabular}


Altura de planta. Los datos del análisis de varianza para variable altura de la planta, presentó diferencias significativas.

Tabla 4 Efectos de la orina humana fermentada (OHF) a los 3, 6 y 12 meses para la altura de planta (cm) en cultivo de lechuga

\begin{tabular}{llllll}
\hline FV & GL & SC & CM & FC & Pr > F \\
\hline \hline Trat. & 3 & 95.61 & 31.81 & 9.25 & $0.0019^{* *}$ \\
Error & 12 & 14.33 & 3.44 & & \\
Total & 15 & 136.95 & & & \\
\hline CV $=13.08 \%$ & & &
\end{tabular}

CV. $=13.08 \%$

El análisis de varianza con respecto a la altura de la planta, presenta diferencias altamente significativas para los tratamientos en estudio con diferentes tiempos de fermentación de la orina humana.

De acuerdo al ANVA se observa que F calculado es de 9.25 que es mayor al $\mathrm{F}$ tabulado de 0.0019. Por tanto, la diferencia de altura de planta entre tratamiento, es altamente significativa.

Figura 1 Altura de planta con la aplicación de orina humana fermentada (OHF) en diferentes tiempos

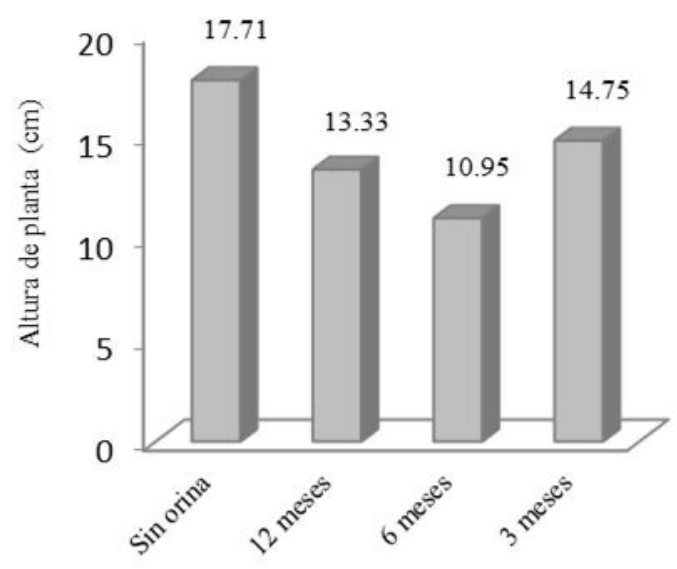

El análisis de la prueba de Duncan al $(\alpha=0.05)$ de probabilidad (figura 1) muestran la formación de tres categorías diferentes: sin orina, se obtuvo un promedio de altura de $17.71 \mathrm{~cm}$, con respecto a los tratamientos con orina fermentada. Por otro lado, la fertilización con 3 y 12 meses de fermentación, se obtuvo similares resultados, en cuanto a la altura de planta, y con 6 meses de fermentación presentó menor altura.

Ancho de hoja. El análisis de varianza para el ancho de la hoja presentó diferencias no significativas, con la aplicación de la orina fermentada en diferentes tiempos, en el cultivo de lechuga (figura 2 y tabla 5).

Figura 2 Ancho de hoja con la aplicación de orina humana fermentada $(\mathrm{OHF})$ en diferentes tiempos

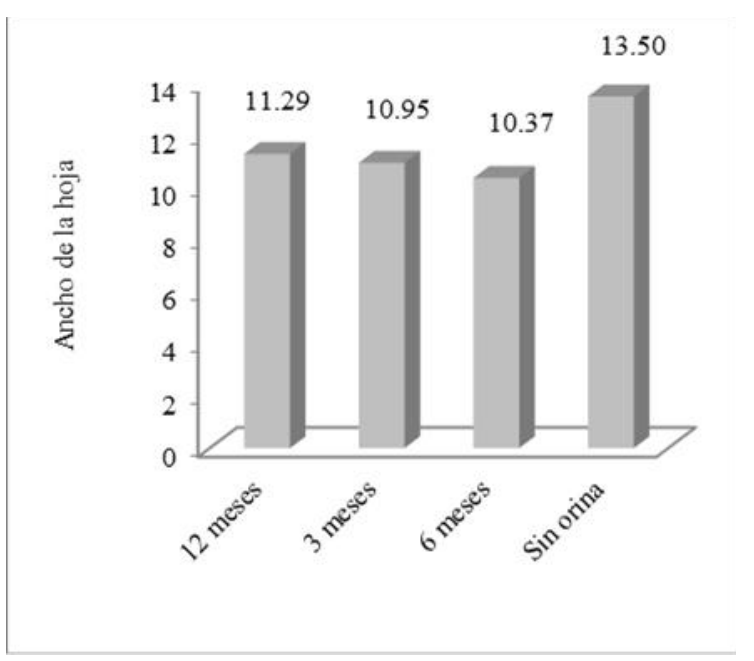

Tabla 5 Efectos de la orina humana fermentada (OHF) a los 3, 6 y 12 meses para ancho de hoja en planta $(\mathrm{cm})$ en cultivo de lechuga

\begin{tabular}{llllll}
\hline FV & GL & SC & CM & FC & Pr $>$ F \\
\hline Trat. & 3 & 20.40 & 7.46 & 2.53 & $0.10 \mathrm{~ns}$ \\
Error & 12 & 35.42 & 2.95 & & \\
Total & 15 & 57.83 & & & \\
\hline CV. $=14.90 \%$ & & & &
\end{tabular}

El análisis de varianza para el ancho de hoja (tabla 5), muestra no significativa para los tratamientos en estudio con diferentes tiempos de fermentación. Asimismo, muestra un coeficiente de variación de 
$14.90 \%$ de valor de confiabilidad y un F calculado de 2.53 y F tabulado de 0.10 .

Longitud de la hoja. Los resultados del análisis de varianza para la longitud de la hoja muestran diferencias significativas.

Tabla 6 Efectos de la orina humana fermentada (OHF) a los 3, 6 y 12 meses para longitud de hoja (cm) en cultivo de lechuga

\begin{tabular}{llllll}
\hline FV & GL & SC & CM & FC & Pr $>$ F \\
\hline \hline Trat. & 3 & 36.73 & 12.24 & 4.03 & $0.033^{*}$ \\
Error & 12 & 36.44 & 3.03 & & \\
Total & 15 & 73.18 & & & \\
\hline CV. $=13.96 \%$ & & & &
\end{tabular}

El coeficiente de variación de $13.96 \%$ obtenido en los tratamientos evaluados con respecto a la longitud de la hoja, se encuentra dentro del rango de los datos confiables.

Figura 3 Longitud de hoja con la aplicación de orina humana fermentada (OHF) en diferentes tiempos

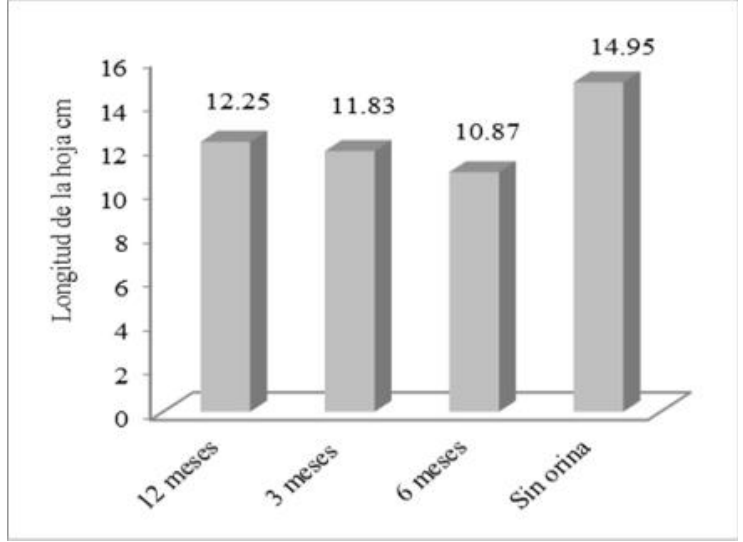

Con respecto a la longitud de la hoja, la prueba Duncan al $\alpha=0.05$ (figura 3), muestra dos grupos. El tratamiento sin orina registró el mayor promedio con $14.95 \mathrm{~cm}$ y con la aplicación de orina de 12 meces de fermentación alcanzó $12.25 \mathrm{~cm}$ y con 3 meses de fermentación de $11.83 \mathrm{~cm}$, siendo el menor valor de $10.87 \mathrm{~cm}$ con 6 meses de fermentación.

Número de hoja por planta. Los resultados del análisis de varianza para el número de hojas, registran diferencias no significativas como se muestra en el (Tabla 7), con la aplicación de la orina fermentada en diferentes tiempos.

Tabla 7 Efectos de la orina humana fermentada (OHF) a los 3, 6 y 12 meses para el numero de hojas en cultivo de lechuga

\begin{tabular}{llllll}
\hline FV & GL & SC & CM & FC & Pr $>$ F \\
\hline \hline Trat. & 3 & 26.06 & 8.68 & 2.72 & $0.910 \mathrm{~ns}$ \\
Error & 12 & 38.31 & 3.19 & & \\
Total & 15 & 64.37 & & & \\
\hline
\end{tabular}

C.V. $=15.58 \%$

El coeficiente de variación obtenida fue de 15.58 $\%$, lo que indica que los datos obtenidos son confiables a los resultados de la evaluación de los tratamientos.

El análisis de ANVA para F calculada es 2.72 y el F tabulado de 0.910 , lo que indica que no existe significancia entre los tratamientos con la fertilización de la orina fermentada.

Figura 4 Número de hojas con la aplicación de orina humana fermentada (OHF) en diferentes tiempos

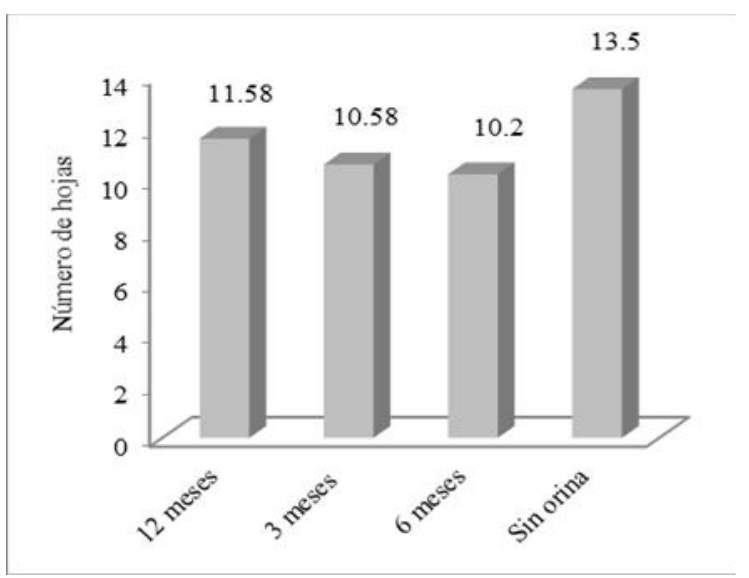


Longitud de la raíz. El análisis de varianza para la diámetro de la raíz, se muestra en la tabla 8, Los valores son altamente significativos entre los tratamientos con la fertilización de orina fermentada.

Tabla 8 Efectos de la orina humana fermentada (OHF) a los 3, 6 y 12 meses para el diámetro de raíz (cm) en cultivo de lechuga

\begin{tabular}{llllll}
\hline FV & GL & SC & CM & FC & Pr $>$ F \\
\hline \hline Trat. & 3 & 2.13 & 0.71 & 0.47 & $0.70 \mathrm{~ns}$ \\
Error & 12 & 18.01 & 1.50 & & \\
Total & 15 & 20.14 & & & \\
\hline \multicolumn{2}{l}{ CV. $=2.72 \%$} & & & &
\end{tabular}

A través del análisis de varianza, se obtuvo un coeficiente de variación de $2.72 \%$, el valor obtenido está en el rango de confiabilidad. También se observa, que F calculado es de 0.47 , que es menor al F tabulada de, 0.70.

Figura 5 Longitud de la raíz con la aplicación de orina humana fermentada (OHF) en diversos tiempos

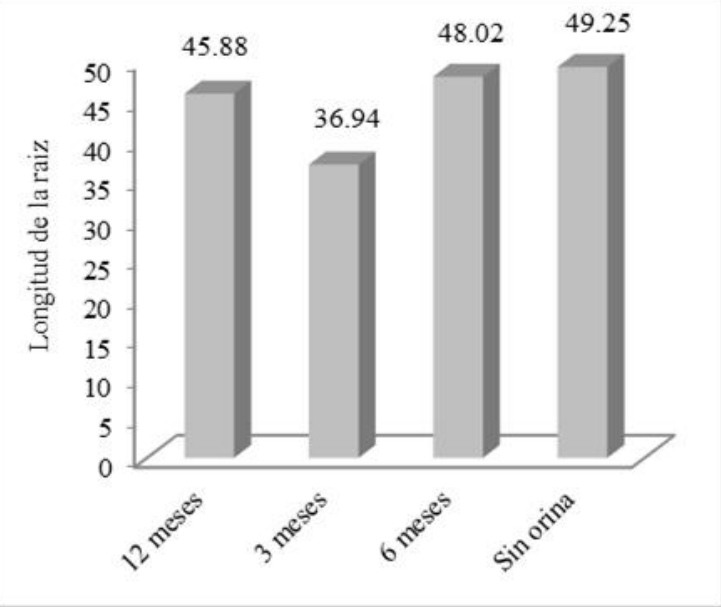

Tabla 9 Efectos de la orina humana fermentada (OHF) a los 3, 6 y 12 meses para el rendimiento en cultivo de lechuga

\begin{tabular}{lcrrrr}
\hline FV & \multicolumn{1}{c}{ GL } & \multicolumn{1}{c}{ SC } & CM & FC & Pr $>$ F \\
\hline \hline Trat. & 3 & 1.93 & 0.64 & 6.5 & 0.0073 \\
Error & 12 & 1.19 & 0.09 & & \\
Total & 15 & 3.12 & & & \\
\hline CV. $=9.00 \%$ & & & &
\end{tabular}

El coeficiente de variación fue de $9.00 \%$, que muestra una alta confiabilidad de los resultados obtenidos sobre el rendimiento. Presenta diferencias no significativas para los tratamientos en estudio (niveles de fertilización). Por tanto, influyó la mayor concentración de nitrógeno de la orina fermentada.

De acuerdo al ANVA (tabla 9), se muestra que F calculada es 6.5, que es mayor al $\mathrm{F}$ tabulada de 0.0073, por tanto, en el rendimiento influyó los diferentes tratamiento aplicados con la orina fermentada.

Figura 6 Rendimiento de lechuga con la aplicación de orina humana fermentada (OHF) en diferentes

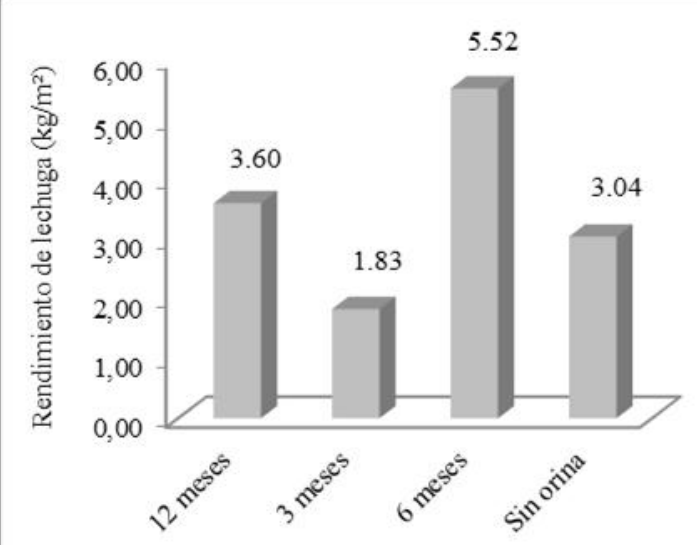


Figura 7 Ingreso neto con aplicación de orina humana fermentada (OHF) en diferentes tiempos

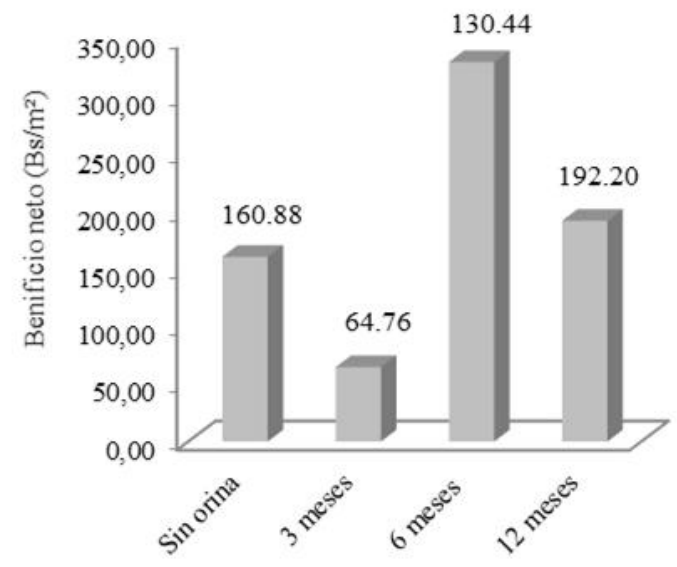

Figura 8 Relación beneficio costo con aplicación de orina humana fermentada (OHF) en diferentes tiempos

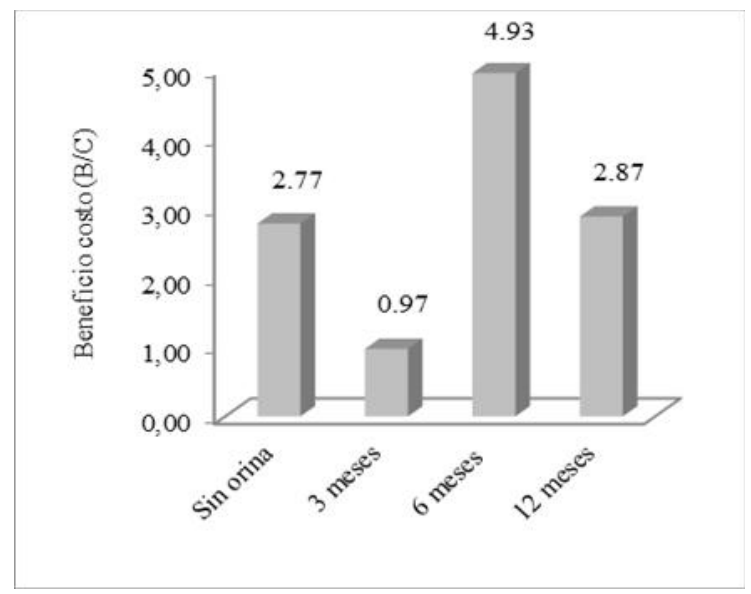

Tabla 10 Detección de Escherichia coli y Salmonella en muestras de lechuga Lactuca sativa L., a 12 meses de fermentación de la orina humana (OHF) aplicada al cultivo

\begin{tabular}{cccc}
\hline Método & Parámetro & $\begin{array}{c}\text { Valor } \\
\text { encontrado }\end{array}$ & $\begin{array}{c}\text { Valor } \\
\text { permitido }\end{array}$ \\
\hline \hline NB-32005 & Escherichia coli & $<1.0 \times 10^{2} \mathrm{UFC} / \mathrm{g}$ & $1 \times 10^{2} \mathrm{UFC} / \mathrm{g}$ \\
NB-32007 & Salmonella spp. & Ausencia en $25 \mathrm{~g}$ & Ausencia en $25 \mathrm{~g}$ \\
\hline
\end{tabular}

Normas de Referencias: Reglamento sanitario alimentos chile/07

\section{Discusión}

La lechuga es un cultivo de clima frío y producirá plantas de mejor calidad en las condiciones frías de invierno y primavera, su crecimiento a temperaturas nocturnas menores a $4^{\circ} \mathrm{C}$ pero en forma lenta. Las temperaturas diurnas no son críticas y la ventilación en sistemas de campo cubiertos con malla e invernaderos debe proveer una temperatura entre los rangos de 12 a $21{ }^{\circ} \mathrm{C}$. (Morgan 1999).

El cultivo de lechuga se desarrolla en ambientes con humedad relativa (HR) 60 a $80 \%$, mayores o inferiores a estos valores, suelen alterar el crecimiento y desarrollo normal del cultivo, favoreciendo la proliferación, el ataque de enfermedades y plagas a los cultivos, cuando supera el $80 \%$ HR. (Vigiola 2005)

Los resultados de la orina fermentada, en relación a la presencia del nitrógeno se puede apreciar que, la fermentación durante 6 meses, presentó mayor contenido de nitrógeno total, con $4026 \mathrm{mg} \mathrm{L}^{-1}$. Cuando la orina se excreta, su pH está generalmente alrededor de 6 , pero puede variar entre $4.5 \mathrm{y}$ 8.2 (Lentner et al. 1981). El 75-90\% del $\mathrm{N}$ es excretado como urea y el porcentaje remanente como amonio y creatinina (Lentner et al. 1981). La ureasa degradada la urea a amonio y dióxido de carbono, los iones hidróxido producidos, normalmente incrementan el $\mathrm{pH}$ a 9-9.3. Normalmente la ureasa se acumula en el sistema de tuberías de la orina y por tanto la transformación antes mencionada es muy rápida, generalmente en cuestión de horas (Vinnerås et al. 1999). Nuestros resultados en relación al pH están en el rango de 9.1 a 9.4 en el año que duro la fermentación, dieron la formación de amonio, permitiendo así su aplicación en los cultivos. 
La disponibilidad del $\mathrm{N}$ de la orina para las plantas es la misma que la de los fertilizantes químicos de urea o amonio, dado que el $90-100 \%$ de $\mathrm{N}$ de la orina se encuentra como urea y amonio (Kirchman \& Pettersson 1995, Richert-Stintzing et al. 2001) El P en la orina es prácticamente (95-100\%) inorgánico y es excretado en forma de iones de fosfato (Lentner et al. 1981). Estos iones están disponibles directamente para las plantas y su disponibilidad para las plantas (Kirchmann \& Pettersson 1995).

La diferencia en altura de la planta se atribuye a la dosificación con mayor concentración de orina, que provocó saturación de nitrógeno causando un retraso en el acogollamiento de la planta. De ahí, la fijación biológica de nitrógeno (FBN), un recurso importante de N para las plantas (Urzúa 2005), que está restringida a los procariontes y la familia de las Rhizobiaceae es la más importante de los organismos que poseen la capacidad de transformar el $\mathrm{N}$ atmosférico en amonio que es asimilado por las fabáceas (Twornlow 2004).

Ibáñez (2004) señala que el crecimiento es un proceso fisiológico muy complicado que depende de otros procesos que tienen lugar en una planta, como la fotosíntesis, reparación, absorción de agua y sustancias minerales.

El análisis de la prueba de Duncan al $(\alpha=0.05)$ de probabilidad (figura 1) muestran la formación de tres categorías diferentes: la primera conforma con la mayor altura de $17.71 \mathrm{~cm}$, siendo superior al resto de los tratamientos, la fertilización de 12 meses y 6 meses de fermentación de la orina son similares en la altura de planta con 14.75 y 13.33 $\mathrm{cm}$, la tercera alcanzo una altura menor $10.95 \mathrm{~cm}$ con aplicación tres meses de fermentación de la orina humana.

La diferencia en altura de la planta puede ser atribuible a la dosificación de orina, en mayor canti33 dad provoco saturación de nitrógeno, causando un retraso en el acogollamiento de la planta. Hartmann (2003), indica que uno de los indicadores del estrés hídrico o desequilibrio hídrico en las plántulas de lechuga, es la pérdida de turgencia y disminución de la tasa de crecimiento, dando como resultado de menor tamaño de órganos de la planta. Fernández (2005) menciona que el agua es un factor importante para el crecimiento y desarrollo de las plantas, desde la germinación hasta la madurez fisiológica de los cultivos, que a lo largo de su crecimiento, el agua proporciona un medio de transporte de elementos nutritivos y la conservación de nutrientes. Moroto (2000), señala que la aplicación de fertilizante orgánico, el uso eficiente de nutrientes desde la germinación de la semilla, hasta la madurez fisiológico de la planta.

El análisis de la prueba de Duncan para el ancho de hoja al $\alpha=0.05$ de probabilidad (Figura 2), fue de $13.50 \mathrm{~cm}$, siendo superior al resto de los tratamientos, la fertilización de 3 y 12 meses de fermentación de la orina, son similares en cuanto al ancho de hoja, con valores de 11.29 y $10.95 \mathrm{~cm}$, a 6 meses de fermentación presentó $10.37 \mathrm{~cm}$ de ancho. La diferencia probablemente se debe a la mayor concentración de nitrógeno en la orina, influyendo en el crecimiento lento de la planta. Rodríguez (1991) señala que existen diferencias en ancho de la hoja, al aplicar diferentes niveles y fermentación de la orina humana, que influye en el crecimiento heterogéneo de la planta. El tratamiento sin fertilización con orina alcanzó un promedio de $13.50 \mathrm{~cm}$. lo que indica que la fertilización se debe practicar en la tercera fase, en la formación de cogollo, para su desarrollo óptimo.

Con respecto a la longitud de la hoja, la prueba Duncan al $\alpha=0.05$ (figura 3), se tiene dos grupos. El tratamiento sin orina registró el mayor prome- 
dio con $14.95 \mathrm{~cm}$ y con la aplicación de orina de 12 meses de fermentación alcanzó $12.25 \mathrm{~cm}$, con 3 meses de fermentación $11.83 \mathrm{~cm}$, siendo el menor valor $10.87 \mathrm{~cm}$ con 6 meses de fermentación. De acuerdo al ANVA (Tabla 6) se observa que el $\mathrm{F}$ calculado es de 4.03 y el $\mathrm{F}$ tabulado es de 0.033 lo que indica que existe significancia entre los tratamiento con la fertilización de orina fermentada. La diferencia que existe en el desarrollo de la longitud de las hojas se debe por la saturación de nitrógeno de orina humana fermentada, que dificultaría la asimilación de otros elementos. Serrano (2006) señala que existen diferencias en longitud de hoja al aplicar diferentes niveles de fermentación de la orina, que presenta con mayor dosis de fertilización orgánica liquido en las plantas de hoja. Por su parte, Oviedo (2001) menciona que el desarrollo de los cultivos requiere ciertos nutrientes para su mayor crecimiento y desarrollo de las plantas.

La (figura 4) mediante el análisis de la prueba de Duncan al $\alpha=0.05$, señala que, en el testigo se obtuvo 13.50, con aplicación de 12 meses de fermentación se tuvo 11.58 hojas, con 3 y 6 meses de fermentación 10.58 y 10.20 hojas, la concentración excesiva de nitrógeno en la orina, influye en el crecimiento y desarrollo lento de la planta. Guerrero (2006) comenta que el nitrógeno es un elemento importante que interviene en la absorción iónica, fotosíntesis, respiración, multiplicación y diferenciación celular, que estimula el aumento del número y tamaño de las células foliares, determinando así el crecimiento vegetativo y reproductivo. La deficiencia de fosforo en la lechuga provoca coloración verde oscuro, y se reduce el desarrollo, las hojas adquieren un aspecto bronceado en los casos más acusados, las plantas no logran acogollar (Díaz 1998).
La prueba de Duncan al 5\% (figura 5) presenta diferencias significativas: en cuanto a la longitud de la raíz sin la aplicación de orina $49.25 \mathrm{~cm}$, con aplicación de 6 meses de fermentación $48.02 \mathrm{~cm}$. Díaz (1998) menciona que existe diferencias entre longitud de la raíz principal y raíces laterales, por aplicación de diferentes dosis de fertilización con orina humana. Cruz (2004) señala que la longitud de la raíz no excede de $0.35 \mathrm{~m}$ de profundidad en cultivo de lechuga, para la cosecha. Guerrero (2006) argumenta que la mayor o menor longitud de raíz está en función a disponibilidad de nitrógeno en el suelo.

El análisis de la prueba de Duncan al $\alpha=0.05$, presenta diferencias estadísticas en cuanto al rendimiento de la lechuga (figura 6). La fertilización con 6 meses de fermentación, alcanzó un rendimiento de $5.52 \mathrm{~kg} \mathrm{~m}^{-2}$, seguido de la fermentación de 12 meses con $3.60 \mathrm{~kg} \mathrm{~m}^{-2}$ de lechuga, el testigo de alcanzó $3.04 \mathrm{~kg} \mathrm{~m}^{-2}$, la fermentación de 3 meses se obtuvo $1.83 \mathrm{~kg} \mathrm{~m}^{-2}$. Hartmann (2003) señala que el objetivo esencial de la fertilización orgánico liquido es asegurar la continuidad de la nutrición nitrogenada a los cultivos, más aun, la lechuga que es una hortaliza de hoja. Marín (2006) indica las fluctuaciones en cuanto al rendimiento, son debidas a los efectos beneficiosos de los distintos abonos orgánicos, que aportan macro y micronutrientes a los cultivos.

De acuerdo a la figura 7, los mayores ingresos netos con la aplicación de 6 meses de fermentación, llegando a Bs. 330.44 por tratamiento, seguido con fertilización de 12 meses de fermentación, obteniéndose Bs. 192.20 por tratamiento, con el testigo Bs. 160.88 por tratamiento, con aplicación de 3 meses de fermentación, se obtuvo un ingreso de Bs. 64.76 por tratamiento. 
En la figura 8, se muestra la relación beneficiocosto $(\mathrm{B} / \mathrm{C})$, obteniéndose una rentabilidad con aplicación de orina humana de 6 meses de fermentación, con beneficio/costo de Bs. 4.93, lo que significa que este tratamiento es bastante rentable, que se puede recomendar su aplicación en el cultivo.

En el testigo el beneficio-costo de 2.77, el tratamiento 4 con aplicación de 12 meses de orina fermentada se obtuvo 2.87 , a 3 meses de fermentación menor beneficio costo. Tampoco, existe ganancia en la producción en el tratamiento 2.

El análisis bromatológico realizado a las lechugas cosechadas, no presentó patógenos de ninguna naturaleza, lo que en conclusión indica que, es completamente inocuo y apto para el consumo humano.

Detección de Escherichia coli y Salmonella en muestras de lechuga, no presentan patógenos, que nos permite apoyar su consumo. Los patógenos entéricos que pueden contaminar la orina es de importancia para evaluar los riesgos de higiene relacionadas con el manejo y uso de la orina como fertilizante. La supervivencia de microorganismos en la orina a través del tiempo de fermentación se ve afectada, estudios realizados con diferentes microorganismos añadidos a la orina y su inactivación seguido en el tiempo (Höglund 2001), a temperatura y $\mathrm{pH}$ elevado cercano a 9 en combinación con amoniaco han concluido a afectar a la inactivación de microorganismos, como Salmonella y E. coli (así como otras bacterias Gramnegativas) fueron inactivados rápidamente. Las bacterias Gram-positivas tenían tasas de inactivación similares y tienen un lento decrecimiento de las bacterias Gram-negativas (Höglund 2001).
Basándose en estos estudios de inactivación de patógenos/indicador en la orina, se han propuesto directrices para el tiempo de almacenamiento de orina y temperatura (Jönsson et al. 2000, Höglund, 2001). Las temperaturas fueron escogidas principalmente en base a las condiciones de clima templado. Las directrices han sido aprobadas por la Agencia de Protección Ambiental de Suecia (EPA), pero aún no se implementa como reglamentos nacionales. Ellos se contabilizarán en las nuevas directrices de la OMS revisadas para el uso de las excretas. Para los hogares individuales, la mezcla de la orina se puede utilizar sin almacenamiento para todo tipo de cultivos, siempre que la cosecha se destina para el consumo propio, que transcurra un mes entre la fertilización y cosecha, es decir, tiempo entre la última aplicación y el consumo.

La agricultura sustentable implica que se requiere un flujo balanceado de nutrimentos e independencia de los fertilizantes, trabajos realizados en campo y laboratorio demuestran que se pueden lograr cosechas semejantes si se aplica orina en lugar de fertilizante mineral. Kirchmann \& Petterson 1995 , reportaron que no existía información disponible acerca del uso de orina humana como fertilizante, solamente la mezcla en agua residual con las heces, Rodhe et al. 2004, reportó que habían pocos estudios de la orina humana como fertilizante en la agricultura, además de un conocimiento limitado de cómo la orina humana trabaja y cómo debe ser manejada, sin embargo, fertilizantes comparables como la orina de animales, que son aplicadas en Suecia para propósitos agrícolas en cantidades aproximadas de 2.3 millones de toneladas por año. En la actualidad se ha incrementado el interés de utilizar la orina humana como fertilizante, debido al crecimiento poblacional e incremento de la de- 
manda de alimentos y los requerimientos para ahorrar agua y energía (Pradhan et al. 2007). Hay reportes que la orina se ha utilizado para fertilizar, de manera experimental, una gran variedad de cultivos, entre los que se encuentran: maíz (Guzha et al. 2005), pepino (Heinonen-Taski et al. 2007, Pradhan et al. 2007), trigo (Tidåker et al. 2007a), jitomate (Pradhan et al. 2009), calabaza (Pradhan et al. 2009a), bamboo (Ndzana \& Otterpohl 2009), beet (Pradhan et al. 2010).

En sí, el uso de la orina como fertilizante en el cultivo de lechuga puede ser utilizada como una fuente alternativa de nutrimentos en la producción de plantas de lechuga, tomado en cuenta el manejo de la orina para que esta, no sea contaminada con materia fecal, desde su recepción hasta su aplicación en los cultivo.

\section{Conflictos de intereses}

Esta investigación recibió financiamiento de la Fundación Sumaj Huasi y apoyo del coordinador del área técnica, para la investigación y no presenta conflictos de interés.

\section{Agradecimientos}

Los autores agradecen al personal Técnico de la Fundación Sumaj Huasi, al Instituto de Ecología, Facultad de Ciencias Puras y Naturales, Universidad Mayor de San Andrés. Al Instituto Nacional de Laboratorio en Salud (INLASA), al Instituto de Investigación y Extensión Agrícola, la Carrera de Ingeniería Agronómica, Universidad Pública de El Alto (UPEA).

\section{Literatura citada}

AdeOluwa OO, Cofie O. Urine as an alternative fertilizer in agriculture: effects in amaranths (Amaranthus caudatus) production. Renew Agr Food Syst. 2012;27(4):287-294.

Díaz R. Aplicación fraccionada de nitrógeno en tres densidades de plantación de lechuga bajo carpa solar. 1998; 70-72 pp.

Fernández G. Capacidad germinativa en especies hortalizas anuales mediante uso de diferentes fertilizantes orgánicos. 2005; 55 pp.

Guadarrama RO, Pichardo NA, Morales-Oliver E. Urine and Compost Efficiency Applied to Lettuce under Greenhouse Conditions in Temixco, Morales, Mexico. In Abstract Volume, First International Conference on Ecological Sanitation 5-8 November 2001, Nanning, China. 2001.

Guerrero J. Abonos orgánicos en tecnología para el manejo ecológico del suelo. Lima Perú. 2006; 42 pp.

Guzha E, Nhapi I, Rockstrom J. An assessment of the effects of human faeces and urine on maize production and water productivity. Phys Chem Earth. 2005; 30: 840-845.

Hartmann F. Invernaderos y ambientes atemperados. FADES. Ed. CECIM. La Paz, Bolivia. 2003; 127 pp.

Heinonen-Tanski H, Sjöblom A, Fabritius H, Karinen P. Pure human urine is a good fertilizer for Cucumbers. Bioresour Technol. 2007;98: 214-217.

Heinonen-Tanski H, Wijk-Sijbesma C. Human excreta for plant procution. Bioresour Technol. 2005; 96: 403-411.

Höglund C. Evaluation of microbial health risks associated with the reuse of source-separated 
human urine. Tesis de doctorado. Royal Institute of Technology (KTH). Stockholm, Sweden. 2001; 87 pp.

Ibañez C. Estrategia de desarrollo sostenible para el municipio de Sorata provincia Larecaja, $\mathrm{La}$ Paz-Bolivia. 2004.

Jönsson H, Vinnerås B, Höglund C, Stenström TA, Dalhammar G, Kirchmann H. Recycling source separated human urine. (Källsorterad humanurin i kretslopp (In Swedish, English summary)). VA-Forsk Report 2000-1, VAV AB, Stockholm, Sweden. 2000.

Kirchman H, Pettersson S. Human urine-Chemical composition and fertilizer use efficiency. Fer Res. 1995; 40:149-154.

Lentner C, Lentner C, Wink A. Units of Measurement, Body Fluids, Composition of the Body, Nutrition. Geigy Scientific tables. CibaGeigy, Basel, Switzerland. 1981.

Marín M. Recolección, tratamiento y aplicación de la orina humana en las plantas para su producción de rendimientos, México. 2006; 67 pp.

Morgan L. 1999. Lechuga, éxito germinación y desarrollo de la plántula. In: Red hidroponía, LaMolina.<http://www.lamolina.edu.pe/facultad/ciencias/hidroponia/boletin1_5/boletin.

Moroto B. 2000. Horticultura para aficionados. Ediciones Mundi España. 2000; 129-130 pp.

Ndzana JE, Otterpohl R. Urine reuse as fertilizer for bamboo plantations. In K. Ashley, D. Mavinic, \& F. Koch (Eds.), International Conference on Nutrient Recovery from Wastewater Streams. IWA Publishing, London, UK. 2009.

Oviedo E. Cultivo de hortalizas en ambiente atemperado Lima Perú. 2001; 23-25 pp.

Pradhan SK, Holopainen JK, Heinonen-Tanski H. Use of human urine and wood ash as fertilizer for red beet (Beta vulgaris) cultivation and its 37 chemical quality. J Agr Food Chem. 2010; 10: 2034-2039.

Pradhan SK, Holopainen JK, Weisell J, HeinonenTanski H. Human Urine and Wood Ash as Plant Nutrients for Red Beet (Beta vulgaris) Cultivation: Impacts on Yield Quality. J. Agric. Food Chem. 2010; 58:2034-2039.

Pradhan SK, Holopainen JK, Weisell J, HeinonenTanski H. Human urine and wood ash as plant nutrients for red beet (Beta vulgaris) cultivation: impacts on yield quality. J Agr Food Chem. 2010; 58 (3):2034-2039.

Pradhan SK, Pitkanen S, Heinonen-Tanski H. Fertilizer value of urine in pumpkin (Cucurbita maxima L.) cultivation. J Agr Food Sci. 2009a; 18:57- 68 .

Pradhan SKJ, Holopainen JK, Heinonen-Tanski H. Store human urine supplementd wich wood ash as fertilizer in tomato (Solanum lycopersicum) cultivation and its impacts on fruit yield and quality. J Agric Food Chem. 2009; 57:76127617.

Pradhan SK, Holopainen JK, Heinonen TH. Stored human urine supplemented with wood ash as fertilizer in tomato (Solanum lycopersicum) cultivation and its impacts on fruit yield and quality. J. Agric. Food Chem. 2009; 57: 76127617.

Pradhan KS, Nerg A, Sjöblom A, Holopainen JK, Heinonen-Tanski H. Use of Human Urine Fertilizer in Cultivation of Cabbage (Brassica oleracea)-Impacts on Chemical, Microbial, and Flavor Quality J Agric Food Chem. 2007; 55: 8657-8663.

Rauch W, Brockmann D, Peters I, Larsen TA, Gujer W. Combining urine separation with waste design: an analysis using a stochastic 
model for urine production. Water Res. 2003; 37(3): 681-689.

Richert A, Gensch R, Jönsson H, Thor-Axel S, Linus D. Guía práctica de uso de la orina en la producción Agrícola. Stockholm: EcoSanRes Programme. Guía, Stockholm Sweden. 2011; $73 \mathrm{pp}$.

Richert-Stintzing A, Rodhe L, Åkerhielm H. Human urine as fertilizer-plant nutrients, application technique and environmental effects (In Swedish, English summary). JTI-Rapport Lantbruk y Industri 278, Swedish Institute of Agricultural and Environmental Engineering. Sweden. 2001.

Rodhe L, Richert Stintzing A, Steineck S. Ammonia emissions after application of human urine to clay soil for barley growth. Nutr Cycl Agroecosys. 2004; 68:191-198.

Rodríguez J. Métodos de investigación pecuaria. Trillas México. 1991; 146-153 pp.

SENAMHI. Departamento de suministro de información hidrometeorológico.Estación Kallutaca (UPEA). 2008. La Paz. Serie Climática: 1967-73/77-08.

Serrano Z. Cultivo de hortalizas en invernadero Editorial Barcelona. Barcelona España. 2006; 33-37 pp.

Shrestha D, Srivastava A, Shakya SM, Khadka J, Acharya BS. Use of compost supplemented human urine in sweet pepper (Capsicum annu$u m$ L.) production. Sci Hort. 2013; 153:8-12.

Thorup-Kristensen K. Root growth and soil nitrogen depletion by onion, lettuce, early cabbage and carrot. Acta Hortic. 2001; 563: 201-206.

Tidåker P, Mattsson B, Jönsson H. Environmental impact of wheat production using human urine and mineral fertilisers - a scenario study. $\mathbf{J}$ Clean Prod. 2007a; 15:52-62.
Twornlow S. 2004. Increasing the role of legumes in smallholder farming systems. The future challenge. In: Rachid Serraj (Ed.) Simbiotic Nitrogen Fixation. Sci Publ Inc. USA. 2004; $382 \mathrm{pp}$.

Urzúa H. Ensayo. Beneficios de la fijación biológica de nitrógeno en Chile. Santiago de Chile. Cienc Inv Agr. 2005; 32:133-150.

Vigiola M. Manual de horticultura. Edición hemisferio Sur. Buenos Aires-Argentina. 2005; 81$88 \mathrm{pp}$.

Vinnerås $\mathrm{B}$, Jönsson $\mathrm{H}$. The performance and potential of faecal separation and urine separation to recycle plant nutrients in household wastewater. Bioresour Technol. 2002; 84(3):275-283.

Vinnerås $B$, Höglund $C$, Jönsson $\mathrm{H}$, Stenström TA. 'Characterisation of sludge in urine separating sewerage systems'. In: Klöve B., Etniner C., Jenssen P. y Maehlum T. (eds). 1999. Proceedings of the $4^{\text {th }}$ International ConferenceManaging the Wastewater Resource Ecological Engineering for Wastewater Treatment. Norway. June 7-11. 1999.

WHO. Guidelines for the safe use of wastewater, excreta and greywater use in agriculture and aquaculture. Socio cultural, environmental and economic Aspects. 3(7). World Health Organisation.2006. (Online access 2014-01-10). www.who.int/sites 